\title{
Dissemination of cognitive behavioral therapy for mood disorder under the national health insurance scheme in Japan: A descriptive study using the National Database of Health Insurance Claims of Japan with special focus on Japan's southwest region
}

\author{
Yuta Hayashi ${ }^{1) \dagger}$, Naoki Yoshinaga ${ }^{2)^{\dagger *}}$, Yuko Yonezawa ${ }^{3)}$, \\ Hiroki Tanoue $^{1)}{ }^{4)}$, Yasuji Arimura ${ }^{3)}$, Kensuke Yoshimura ${ }^{5)}$, \\ Toshihiko Yanagita ${ }^{3)}{ }^{4)}$, Keiko Aoishi ${ }^{6)}$, Yasushi Ishida ${ }^{7)}$
}

\begin{abstract}
Cognitive therapy/cognitive behavioral therapy (CBT) has consistently been shown to be effective for a range of mental disorders, and was introduced into psychiatric care in Japan in the late 1980s. Since April 2010, the national health insurance scheme in Japan has covered CBT for mood disorders, though its utilization status remains empirically unknown. The current descriptive study aimed to assess the dissemination status of CBT for mood disorders in the first six years (FY2010-2015), using the National Database of Health Insurance Claims of Japan, with special focus on the situation in the Kyushu region, located in the southwest of Japan. Results revealed that the numbers of CBT sessions and patients who received CBT followed different trends between FY2010 and FY2015 in this region compared to overall utilization throughout Japan. The share of CBT sessions and patients in the Kyushu region compared to nationwide utilization showed an increasing trend from FY2010 to FY2012, but thereafter tended to decrease $(p<0.0001$, respectively). These changes may reflect trends in Kumamoto prefecture, which initially had the far largest number of CBT sessions per registered institution in the region, but then drastically decreased after FY2013. The number of institutions registered to provide CBT per 100,000 population increased only slightly over time throughout the entire Kyushu region, with small prefectural variations. Further detailed analysis may be required to reveal the factors that influence the dissemination process of CBT in Japan overall and in local regions.
\end{abstract}

[Key words] Cognitive behavioral therapy, mood disorder, depressive disorder, national claims database, Kyushu, Japan.

1) Graduate School of Medicine and Veterinary Medicine, University of Miyazaki, Japan.

2) Organization for Promotion of Tenure Track, University of Miyazaki, Japan.

3) Clinical Research Support Center, University of Miyazaki Hospital, Japan.

4) School of Nursing, Faculty of Medicine, University of Miyazaki, Miyazaki, Japan.

5) Department of Health and Welfare Services, National Institute of Public Health, Japan.

6) Department of Nursing, Graduate School of Medicine, Nagoya University, Japan.

7) Division of Psychiatry, Department of Clinical Neuroscience, Faculty of Medicine, University of Miyazaki, Japan.

$\dagger$ Contributed equally

* Corresponding author 


\section{Introduction}

Effective and accessible treatments for psychiatric disorders are in high demand worldwide. According to a WHO report, unipolar depression will be the leading cause of disease burden in high-income countries by 2030, and will be a major burden following HIV/AIDS and perinatal disorders in low-income and middle-income countries. ${ }^{1}$ Over the last 40 years, considerable progress has been made in developing effective, evidence-based psychological interventions for a wide range of psychiatric disorders. Among them, forms of cognitive therapy/ cognitive behavioral therapy (CBT) have consistently been proven to be effective for a range of mental disorders, ${ }^{2}$ and to exhibit higher efficacy than pharmacotherapy in treating some disorders ${ }^{3,4} \mathrm{CBT}$ is now a highly recommended treatment modality for several mental disorders in many national guidelines including those in the UK, USA, Canada, Australia, and Japan. ${ }^{5-9}$ However, CBT is too often delivered suboptimally in routine clinical practice..$^{10,11}$ CBT was introduced into the field of psychiatry in Japan in the late $1980 \mathrm{~s} .{ }^{12}$ CBT is based on the principle that thoughts and perceptions can impact feelings and behaviors, and that individuals can learn flexible, realistic, adaptive ways of thinking that will subsequently influence their behaviors through cognitive (e.g. cognitive restructuring) and behavioral (e.g. exposure, behavioral experiments, social skills training, relaxation training) techniques. ${ }^{13}$ Numerous studies in Western countries ${ }^{14,15}$ and most recently in Japan ${ }^{16-18}$ have demonstrated the efficacy of CBT for depression on both a short- and long-term basis. With the accumulation of evidence, the national health insurance scheme in Japan began to cover CBT for mood disorders from April 2010. This marked a milestone in the provision of mental health services in this country where pharmacotherapy has historically been the far more common approach. ${ }^{19-21}$ Moreover, depressed primary care patients often express the wish to receive psychotherapy rather than pharmacotherapy. ${ }^{22}$

However, patient access to appropriate CBT services was reportedly very limited in Japan. ${ }^{23-25}$ According to a nationwide survey conducted in 2006 , only $28 \%$ of all medical facilities were capable of providing any form of psychotherapy in a satisfactory manner. ${ }^{26}$ It was argued that insufficient provision of CBT may have resulted from an insufficient number of CBT providers who had only limited opportunities to receive proper training, and whose reimbursement for CBT under the current national fee schedule was small (e.g. a maximum of 5,000 JPY for one session [equal to $45.5 \mathrm{USD}$, exchange rate: 1 USD $=110 \mathrm{JPY}$ ]). ${ }^{23-25}$ However, no survey studies have been able to establish how often and for how many patients CBT for mood disorder has actually been provided in Japan, especially after FY2010 when CBT for mood disorders first became eligible for coverage under the national health insurance scheme. Assessing the dissemination process of CBT could provide information for policy makers with the objective of finding suitable leverage points to improve patient access to CBT services across Japan; e.g. the development of more effective training programs, further revision of medical service fees, and/or advocacy for required funding and organizational support.

The current descriptive study, using the National Database of Health Insurance of Japan, aimed to report on the actual dissemination status of CBT for mood disorders in the first six years since coverage under national health insurance began. We focus specifically on the situation in the Kyushu region, located in the southwest of Japan where, as is described shortly, the dissemination patterns were distinctly different compared to the national trend, and which may open a window that allows the factors influencing the process of CBT dissemination to be properly investigated.

\section{Methods}

\section{(1) Data collection}

\section{a) Number of CBT sessions}

Two types of CBT for mood disorders have been included in the national health insurance scheme: "CBT (1)," 
which is provided by a designated psychiatrist (Mental Health and Welfare Law-authorized) who also cooperates with psychiatric emergency medical services, and "CBT (2)," which is provided by a medical doctor who does not meet the above criteria for "CBT (1)." The medical fees for "CBT (1)" and "CBT (2)" are 5,000 JPY and 4,200 JPY (equal to 45.5 USD and 38.2 USD), respectively. In both cases, in order to receive reimbursement under the national health insurance scheme, clinicians are required to have completed adequate CBT training (e.g. organized by Japan's Ministry of Health Labour and Welfare), to provide over 30 minutes of individual CBT per session (up to total 16 sessions), and to adhere to the CBT treatment manual for depression. ${ }^{27}$

Numbers of CBT sessions and patients who received CBT (at least one session) were obtained from the National Database of Health Insurance Claims of Japan (NDB). The NDB was developed by Japan's Ministry of Health Labour and Welfare in April 2011, and approximately 1.6 billion claims have been added annually to the NDB. The NDB covers all electronically submitted claims (99\% of all claims issued from medical institutions with $\geq 20$ beds and clinics).$^{28}$ The claims data include clinical and procedural information, such as institution identification numbers, prefectural code of institutions, sex, age, date of admission, date of discharge, procedural codes, diagnostic codes, and drug codes. Personally identifiable data in the NDB (e.g. name, beneficiary identification number, date of birth) are irreversibly encrypted using hash functions. Claims data from FY2010 to FY2015 were included in the current study.

\section{b) Number of institutions registered to provide CBT in the Kyushu region}

Since April 2012, medical institutions have been required to meet specific criteria in order to provide CBT. Institutional registration was managed by each Regional Bureau of Health and Welfare of Japan. In this study, lists of institutions registered to provide CBT in the Kyushu region from FY2012 to FY2015 were obtained from the Kyushu Regional Bureau of Health and Welfare.

\section{(2) Analysis}

The number of CBT sessions throughout Japan and in the Kyushu region, and the number of institutions registered to provide CBT in the Kyushu region were obtained for each fiscal year. The number of CBT sessions was defined as the sum of CBT (1) and CBT (2). The number of institutions registered to provide CBT per 100,000 population and the number of CBT sessions per registered institution in the Kyushu region were also estimated. Furthermore, the share of CBT sessions and patients in the Kyushu region compared to nationwide utilization was calculated, and trends over time were tested using the Cochran-Armitage test. Since descriptive statistics from the first year (FY2010) to the year for which most statistics were available (FY2012) and afterwards were different, the trend was tested before and after FY2012. The tests were two-tailed, and $p$-values of less than 0.05 were considered statistically significant. Since this is an exploratory descriptive study, we did not consider multiplicity issues. The analyses were performed with RStudio 1.1 (RStudio Inc., Boston, Massachusetts, USA), which is an integrated development environment for R (The R Foundation for Statistical Computing, Vienna, Austria).

\section{(3) Ethical considerations}

The study protocol was reviewed and approved by the Ethics Committee of the University of Miyazaki (reference number: O-0017). We also adhered to guidelines on the use of the NDB, according to which we were obligated to access the dataset only in a pre-specified secure room. After a review of this study protocol by the NDB expert council, we entered into a contract with the ministry to use a dataset extracted from the NDB for the purpose of the present study (reference number: 1025-1). Under the conditions of the contract, cell observations fewer than 10 were masked in order to prevent unwanted identification of personal information and medical institutions. Written informed consent was waived because all patient records were automatically anonymized prior to storage in the NDB. 


\section{Results}

A total of 60,304 patients who received 256,423 sessions of CBT were recorded in the database from FY2010 to FY2015. Figure 1 shows trends over time in both Japan overall and the Kyushu region. In Japan, the number of CBT sessions and patients were highest in the first year (FY2010), and reached a nadir in FY2012, followed by a slight recovery after FY2013. In the Kyushu region, the numbers of CBT sessions were almost the same during the first four years, followed by a sharp decrease in FY2014 and FY2015. The numbers of patients were almost the same in the first five years, but decreased in FY2015. Figure 2 shows the share of CBT sessions and patients in the Kyushu region compared to nationwide utilization. There was an increasing trend from FY2010 to FY2012 $(p<0.0001)$, and a decreasing trend from FY2012 to FY2015 $(p<0.0001)$, for both CBT sessions and patients who received CBT. The findings indicated that the pattern of CBT dissemination in the Kyushu region was uniquely different from that in Japan as a whole.

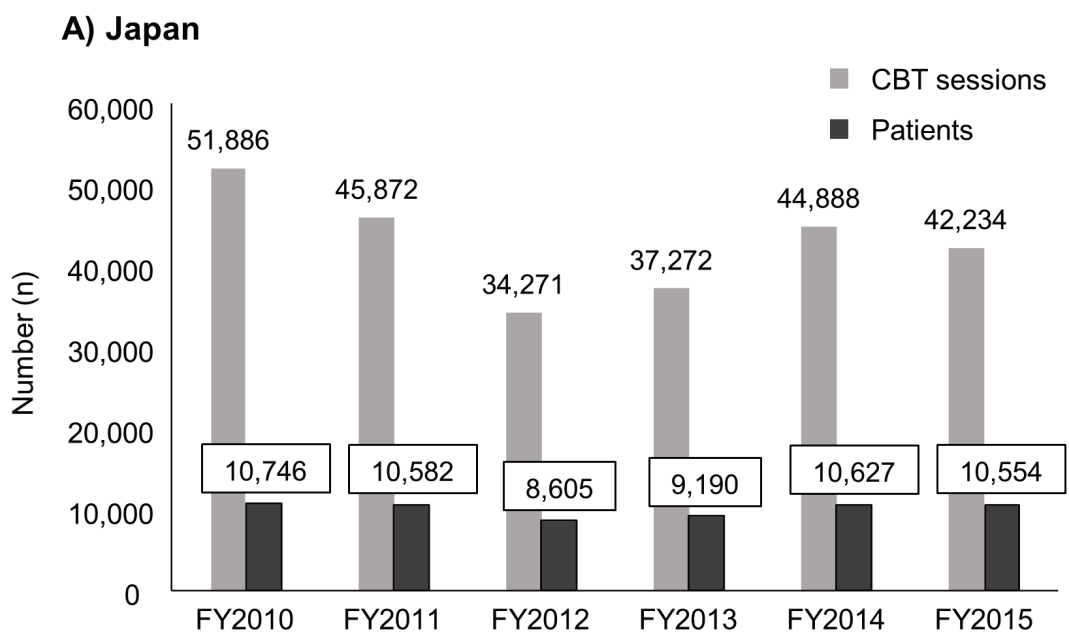

\section{B) Kyushu}

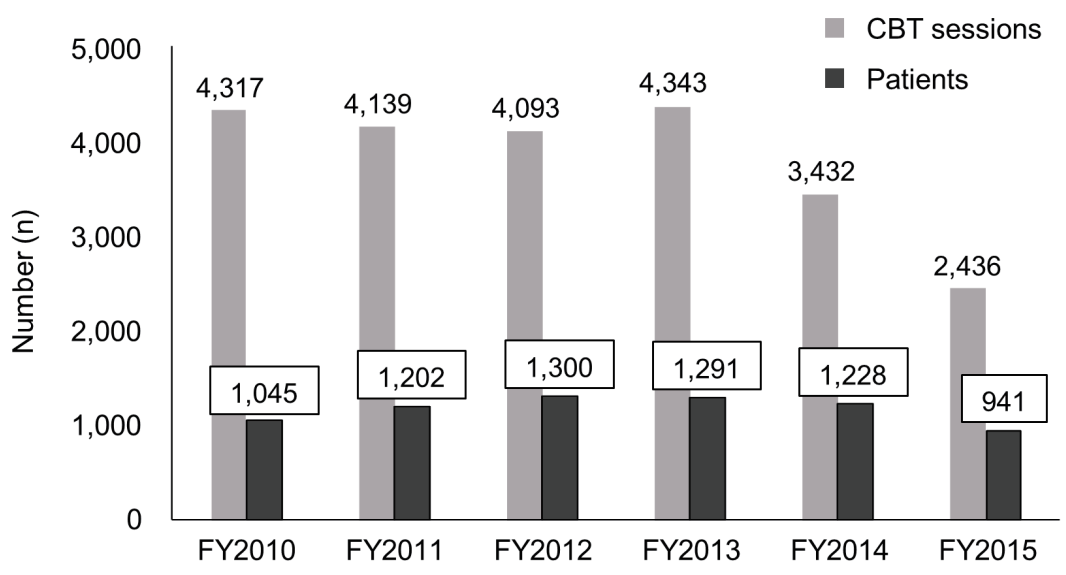

Figure 1. Numbers for provision of CBT in Japan and Kyushu region Note: numbers less than 10 in Kyushu region were not counted. Abbreviation: CBT, Cognitive Therapy/Cognitive Behavioral Therapy. 


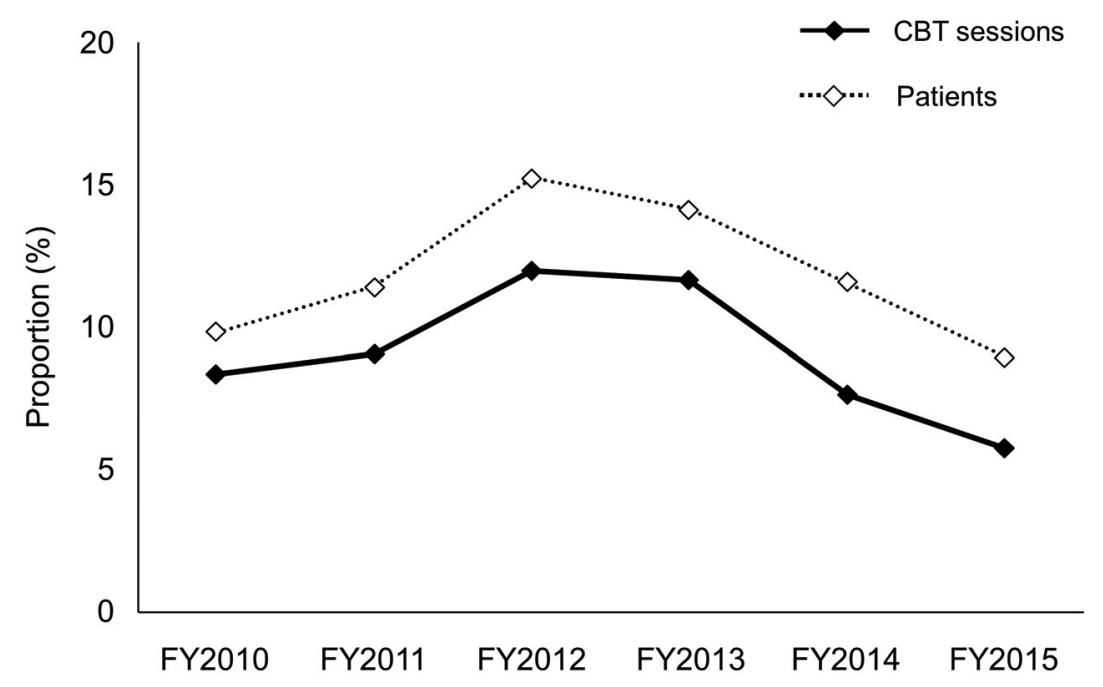

Figure 2. Share of $\mathrm{CBT}$ sessions and patients who received $\mathrm{CBT}$ in the Kyushu region compared to total utilization in Japan Abbreviation: CBT, Cognitive Therapy/Cognitive Behavioral Therapy.

The total number of registered institutions per 100,000 population in the Kyushu region increased by $16.0 \%$ from FY2012 to FY2015 (Figure 3), however, there were observed variations across prefectures, and no consistent trends across prefectures were identified.

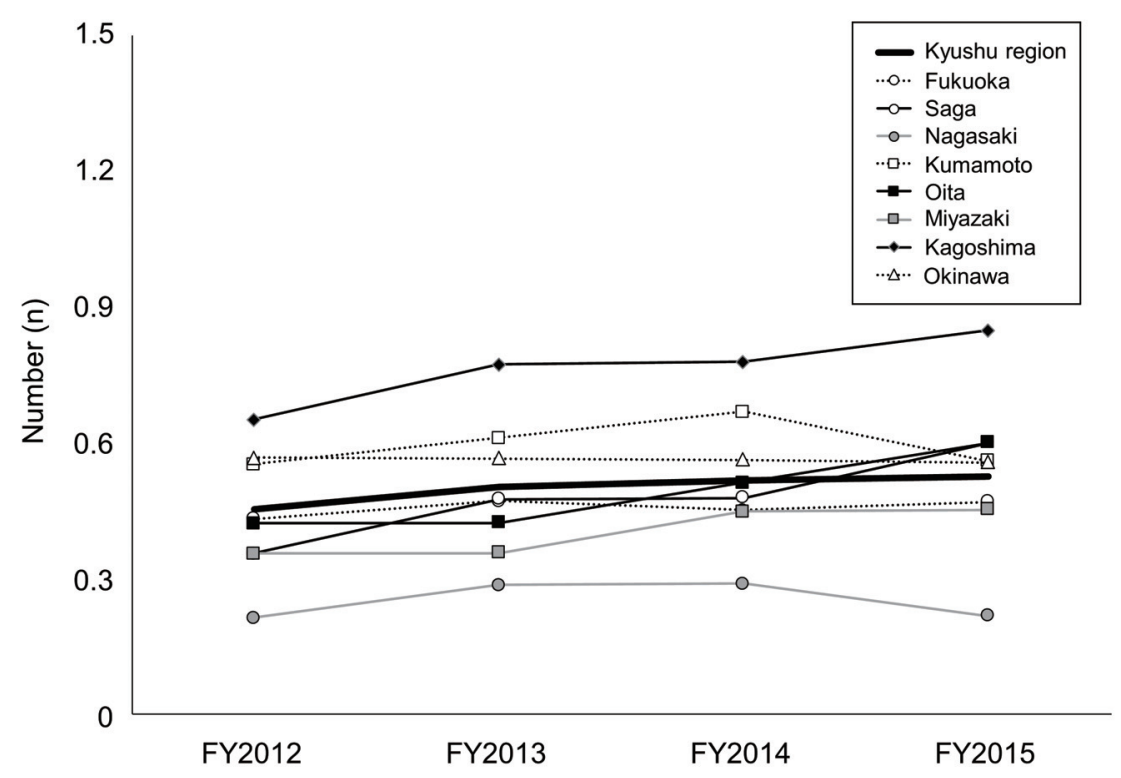

Figure 3. Trend for institutions registered to provide CBT per 100,000 population in Kyushu region Abbreviation: CBT, Cognitive Therapy/Cognitive Behavioral Therapy. 
The number of CBT sessions per institution registered to provided CBT in the Kyushu region as a whole decreased by $42.5 \%$ from FY2012 to FY2015 (Figure 4). Prefectural specific observations revealed that five prefectures (Fukuoka, Nagasaki, Kumamoto, Oita, and Okinawa) showed a decreasing trend over time, most noticeably in Kumamoto where the number of CBT sessions per institution provided in the first two years was about four-fold higher than the Kyushu region average, then dropped close to the average in FY2015. In contrast, a clearly increasing trend was observed in Miyazaki prefecture.

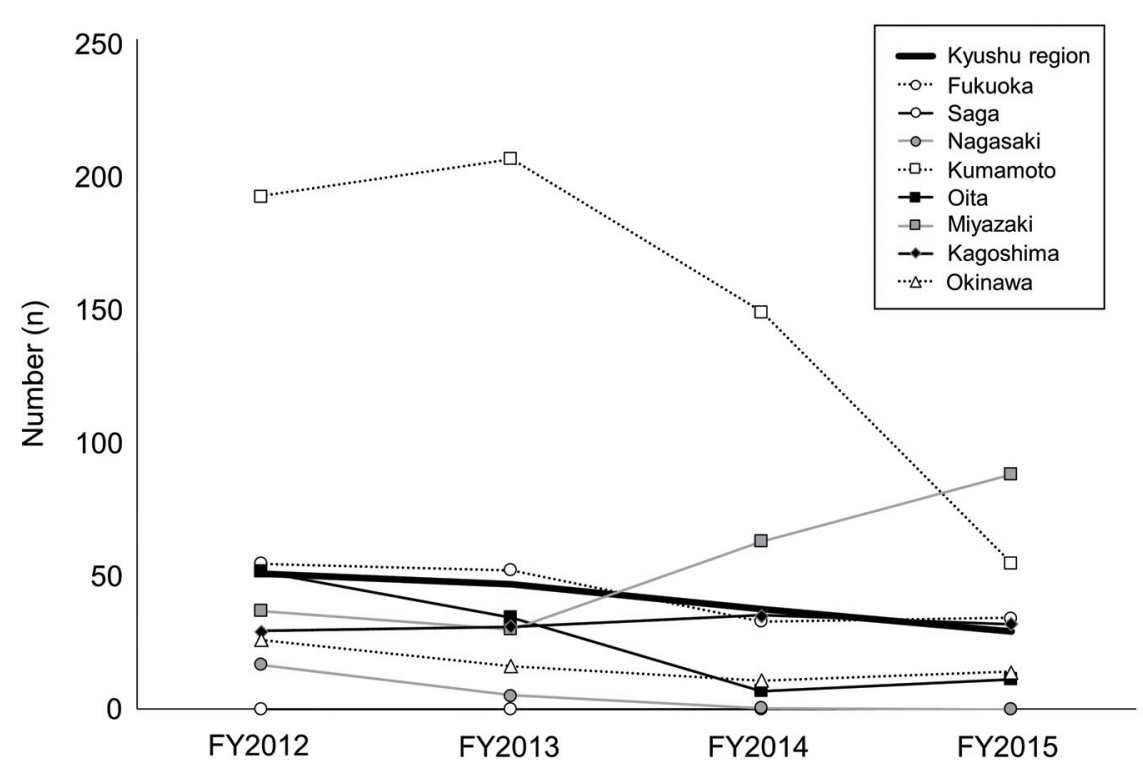

Figure 4. Trend of CBT sessions per registered institution in Kyushu region Note: numbers less than 10 in the original raw data were handled as zero. Abbreviation: CBT, Cognitive Therapy/Cognitive Behavioral Therapy.

\section{Discussion}

This descriptive study is the first to demonstrate the status of CBT dissemination in Japan, and specifically in the Kyushu region, using the national claim-based data. Our results show that up until FY2012 both the number of CBT sessions and patients who received CBT in Japan nationwide dropped after the inclusion of CBT in the national health insurance scheme, then showed a recovering trend thereafter. Compared to the national trend, the Kyushu region had a unique utilization pattern where the drop was observed only after FY2013, and main contributor to this different trend was a single prefecture, Kumamoto, which had the largest number of CBT sessions per institution in the Kyushu region. A noticeable increase in terms of the number of CBT sessions per registered institution was observed only in Miyazaki prefecture.

Clinicians have claimed that the major barrier to CBT dissemination was limited opportunities for formal training to obtain CBT certification that would enable them to provide this therapy under the national health insurance scheme (only two to five times/sites per year throughout Japan). ${ }^{24,29}$ In the Kyushu region, formal CBT training (organized by Japan's Ministry of Health Labour and Welfare) has been held just three times and only in Fukuoka prefecture during the study period (FY2012, FY2014, and FY2015). However, as our results convincingly showed, the numbers of registered institutions per 100,000 population and CBT sessions per registered institution 
in Fukuoka prefecture were almost the same as the average for the entire Kyushu region. Therefore, limited access to formal CBT training session does not explain the CBT dissemination in the Kyushu region during the study period.

Another barrier to CBT dissemination may be insufficient reimbursement for service provision under tight price regulation in the national insurance fee schedule. Psychiatrists can claim fees for " $C B T$ " under the health insurance scheme for at most two patients an hour (i.e. a maximum of 10,000 JPY per hour [equal to 90.1 USD]). On the other hand, in their regular practice, psychiatrists typically can see six patients on average per hour and claim fees for "ambulatory psychotherapy" for each patient (i.e. a maximum of 19,800 JPY per hour [equal to 180 USD]). Therefore, the current fee schedule created a disincentive for clinicians and medical institutions to claim fees for "CBT" rather than "ambulatory psychotherapy." There is also a possibility that clinicians may actually provide CBT in brief sessions ( $<30$ minutes) and claim fees for "ambulatory psychotherapy." Thus, our observation of a decreasing trend in the provision of CBT may be distorted by upcoding in the claim process. Further questionnaire surveys and analyses of data in other regions would be of great assistance to investigate underlying factors that determined the pattern of CBT dissemination in Japan as well as in local regions.

The main limitation of this study is that the NDB used in this study does not cover medical treatment provided in private practice. There are many medical institutions in which CBT is provided in private practice out of national health insurance coverage (e.g. 10,000 JPY [equal to $90.1 \mathrm{USD}$ ] for one session at Chiba University Hospital, Japan ${ }^{30}$ ) due to the low reimbursement rate for CBT in Japan (e.g. Japan, 5,000 JPY per session; in contrast, UK, 97 GBP per session [equal to 14,550 JPY and 132 USD] ${ }^{31}$ ). Further, Sato and Tanno reported that CBT for depression in Japan is mostly delivered by psychologists in routine clinical practice, ${ }^{32}$ but it is not covered by the health insurance scheme. Thus, there is a possibility our estimation, which solely based on the NDB, underestimates the real extent of CBT provision in Japan. This limitation means that a supplementary questionnaire survey should form part of future research.

In conclusion, the present study clearly showed that the number of CBT sessions and patients who receive CBT followed a different trend in the Kyushu region compared to Japan overall after the inclusion of CBT in the national health insurance scheme. The trend in the Kyushu region was heavily influenced by the experience in Kumamoto prefecture which had the largest number of CBT sessions per institute, rather than the number of institutes per se, in the region. Comparative analysis across prefectures in the region indicated that limited access to formal training for reimbursement certification was less likely to be a barrier to CBT dissemination. Further questionnaire surveys and analyses of data in other regions are required to gain a more in-depth understanding of the dissemination status of CBT and the effects of disincentives arising out of price regulation mandated by the national fee schedule.

\section{Acknowledgements}

This study was supported by a FY2017 (21th) research grant for young researchers from the Japanese Institute for Health Economics and Policy (to NY). The authors would like to express their gratitude to Mr. Richard White for checking the English; Ms. Chieko Fujiyama for supporting data collection and entry.

\section{The date of submission}

January 19th, 2018 


\section{References}

1) Mathers, C.D., Loncar, D. Projections of global mortality and burden of disease from 2002 to 2030. PLoS Med. 2006;3:e442.

2) Butler, A.C., et al. The empirical status of cognitive-behavioral therapy: a review of meta-analyses. Clin Psychol Rev. 2006;26:17-31.

3) Sava, F.A., et al. Cost-effectiveness and cost-utility of cognitive therapy, rational emotive behavioral therapy, and fluoxetine (Prozac) in treating depression: a randomized clinical trial. J Clin Psychol. 2009;65:36-52.

4) Mavranezouli, I., et al. The Cost effectiveness of psychological and pharmacological interventions for social anxiety disorder: a model-based economic analysis. PLoS One. 2015;10:e0140704.

5) National Institute for Clinical Excellence (NICE). Depression in adults: recognition and management. NICE guidelines [CG90]; 2009. Available from: http://www.nice.org.uk/guidance/cg90. Accessed December 20, 2017.

6) American Psychiatric Association (APA). Practice guideline for the treatment of patients with acute stress disorder and posttraumatic stress disorder; 2004. Available from: http://psychiatryonline.org/content.aspx?bookid=28\&sectionid=1670530. Accessed December 20, 2017.

7) Parikh, S.V., et al. Canadian Network for Mood and Anxiety Treatments (CANMAT) 2016 Clinical Guidelines for the Management of Adults with Major Depressive Disorder: Section 2. Psychological Treatments. Can J Psychiatry. 2016;61:524-539.

8) Hay, P., et al. Royal Australian and New Zealand College of Psychiatrists clinical practice guidelines for the treatment of eating disorders. Aust N Z J Psychiatry. 2014;48:977-1008.

9) Japanese Society of Mood Disorders. Guideline for treatment of major depressive disorder; 2016. Available from: http://www.secretariat.ne.jp/jsmd/mood_disorder/img/160731.pdf. Accessed December 20, 2017. [in Japanese]

10) Shafran, R., et al. Mind the gap: Improving the dissemination of CBT. Behav Res Ther. 2009;47:902-909.

11) Gunter, R.W., Whittal, M.L. Dissemination of cognitive-behavioral treatments for anxiety disorders: Overcoming barriers and improving patient access. Clin Psychol Rev. 2010;30:194-202.

12) Ono Y, Furukawa TA, Shimizu E, Okamoto Y, Nakagawa A, Fujisawa D, et al. Current status of research on cognitive therapy/cognitive behavior therapy in Japan. Psychiatry Clin Neurosci. 2011;65:121-129.

13) Beck, A.T., et al. Cognitive therapy of depression. New York: Guilford Press, 1979.

14) Wiles, N., et al. Cognitive behavioural therapy as an adjunct to pharmacotherapy for primary care based patients with treatment resistant depression: results of the CoBalT randomised controlled trial. Lancet. 2013;381:375-384.

15) Cuijpers, P., et al. A meta-analysis of cognitive-behavioural therapy for adult depression, alone and in comparison with other treatments. Can J Psychiatry. 2013;58:376-385.

16) Sado, M., et al. Cost-effectiveness of combination therapy versus antidepressant therapy for management of depression in Japan. Aust N Z J Psychiatry. 2009;43:539-547.

17) Fujisawa, D., et al. Cognitive behavioral therapy for depression among adults in Japanese clinical settings: a single-group study. BMC Research Notes. 2010;3:160.

18) Nakagawa, A., et al. Effectiveness of supplementary cognitive-behavioral therapy for pharmacotherapy-resistant depression: a randomized controlled trial. J Clin Psychiatry. 2017;78:1126-1135.

19) International Narcotics Control Board. Report of the International Narcotics Control Board on the availability of internationally controlled drugs: ensuring adequate access for medical and scientific purposes; 2010. Available from: https:/www.incb.org/documents/Publications/AnnualReports/AR2010/Supplement-AR10_availability_English.pdf. Accessed December 20, 2017. [in Japanese]

20) Yoshio, T. The trend for megadose polypharmacy in antipsychotic pharmacotherapy: a prescription survey conducted by the Psychiatric Clinical Pharmacy Research Group. Seishin Shinkeigaku Zasshi (Psychiatr Neurol Jpn). 2012; 114:690695. [in Japanese]

21) Okumura, Y., Shimizu, S., Matsumoto, T. Prevalence, prescribed quantities, and trajectory of multiple prescriber episodes 
for benzodiazepines: A 2-year cohort study. Drug Alcohol Depend. 2016;158:118-125.

22) Dwight-Johnson, M., et al. Treatment preferences among depressed primary care patients. J Gen Intern Med. 2000;15:527-534.

23) Ono, Y. Cognitive therapy/cognitive behavioral therapy in Japan: past, present, future. Jpn J Cogn Ther. 2011;4:1-7. [in Japanese]

24) Kobori, O., et al. Transporting Cognitive Behavioral Therapy (CBT) and the Improving Access to Psychological Therapies (IAPT) project to Japan: preliminary observations and service evaluation in Chiba. J Ment Health Train Educ Pract. 2014;9:155-166.

25) Yoshinaga, N., et al. Cognitive behavioral therapy in psychiatric nursing in Japan. Nurs Res Pract. 2015;2015:529107.

26) Fujisawa, D., et al. Current Status and Dissemination of Psychotherapies in Japan. Tokyo: Health and Welfare Statistics Association, 2006. [in Japanese]

27) Japanese Ministry of Health, Labour and Welfare. CBT Manual for Depression: A Therapist's Manual; 2010. Available from:

http://www.mhlw.go.jp/bunya/shougaihoken/kokoro/dl/01.pdf. Accessed December 20, 2017. [in Japanese]

28) Japanese Ministry of Health, Labour and Welfare. Status of dissemination of electronically submitted claims; 2016. Available from:

http://www.mhlw.go.jp/file/06-Seisakujouhou-12400000-Hokenkyoku/0000099002.pdf. Accessed December 20, 2017. [in Japanese]

29) Center for the Development of Cognitive Behavior Therapy Training. Workshop of Cognitive Behavior Therapy for Depression; 2017. Available from: http://cbtt.jp/studyandtraining/. Accessed March 12, 2018. [in Japanese]

30) Chiba University. Press Release: Chiba University established the first "Cognitive Behavioral Therapy Center" as a National University Hospital in Japan; 2016. Available from: https:/prtimes.jp/main/html/rd/p/000000128.000015177. html. Accessed March 12, 2018. [in Japanese]

31) Curtis, L. \& Burns, A. Unit Costs of Health and Social Care 2016, Personal Social Services Research Unit. Canterbury: University of Kent, 2016.

32) Sato, H., Tanno, Y. The effect of cognitive behavioral therapy for depression delivered by Japanese psychologists: a systematic review. Jpn J Behav Ther. 2012;38:157-167. [in Japanese]

\section{Correspondence}

Naoki Yoshinaga, Lecturer, Organization for Promotion of Tenure Track, University of Miyazaki, General Education and Research Building (G704), 5200 Kihara, Kiyotake, Miyazaki 889-1692, Japan. Tel/Fax: (+81)98585-9784 E-Mail: naoki-y@med.miyazaki-u.ac.jp 